\title{
Palladio Museum: historia y museografía para un nuevo museo- taller sobre la arquitectura de Andrea Palladio
}

El nuevo Palladio Museum, ubicado en el Centro Internazionale di Studi di Architecttura Andrea Palladio de Vicenza, ha iniciado su andadura con una tipología museológica muy novedosa y completamente dedicado a la arquitectura de Andrea Palladio. El allestimento (proyecto expositivo) propone una nueva tipología de museo-taller donde se une la valorización de la arquitectura con la propia investigación en curso sobre ella por parte de un centro de investigación con experiencia de más de medio siglo en la valorización y comunicación de la arquitectura ligada a Andrea Palladio.

Carlos Plaza | arquitecto

URL de la contribución <www.iaph.es/revistaph/index.php/revistaph/article/view/3429>

En octubre de 2012 abrió sus puertas el Palladio Museum en la ciudad italiana de Vicenza, concretamente en la histórica sede del Centro Internazionale di Studi di Architettura (CISA) Andrea Palladio. El nuevo museo se ubica en el palacio Barbaran da Porto, el único de los proyectados por Andrea Palladio que pudo dirigir por completo su construcción y supervisar él mismo su aparato decorativo. El innovador proyecto museográfico está basado en la experiencia pluridecenal del centro en cuanto a investigación en torno a la obra del arquitecto italiano y su puesta en valor ante la sociedad, no como un ejercicio de retórica mitificación de un héroe o de una cultura local, sino como un viaje hasta el interior de todo lo que rodea la arquitectura del gran arquitecto véneto.

\section{Un museo-taller para la investigación y la difusión}

Desde hace más de medio siglo, el CISA Andrea Palladio destaca como centro de investigación en arquitectura con especial interés por la historia de la arquitectura, reuniendo en torno a él, como premisa fundacional, a un comité científico caracterizado por la amplia presencia internacional. En lo que respecta al más importante de entre los intereses del centro, la investigación palladiana, se caracteriza por una investigación a través de los instrumentos de la filología y con gran atención a los contextos y a todo lo que rodea la creación arquitectónica, con el objetivo último de aumentar el conocimiento que poseemos sobre ella y su posterior interpretación, natural premisa para la conservación y la valorización de la arquitectura del pasado. El proyecto museográfico ha sido diseñado por el propio centro a través del presidente de su comité científico, el profesor Howard Burns, y por su director, Guido Beltramini, habiendo sido cuidado el diseño expositivo por el arquitecto Alessandro Scandurra.

La convicción de que la historia de la arquitectura es fruto de la investigación y el trabajo interpretativo de los estudiosos es una cuestión que está en la base del proyecto museográfico. La permanente mutabilidad debido a los avances en el conocimiento está en amplio contraste con una idea de la historia como ciencia generadora de verdades que se consolidan en el tiempo y generan interpretaciones estáticas de los hechos históricos, de sus protagonistas, de sus obras arquitectónicas, etc. Será, por lo tanto, dicha investigación en torno a la arquitectura de Andrea Palladio el principal fondo que subyace entre los objetos expuestos y la propia arquitectura palladiana del Palladio Museum. Es por ello que el proyecto expositivo insiste en la idea de que la exposición es un continuo work in progress interpretativo, e incluso las diferentes secciones son explicadas en realidad virtual por los principales estudiosos del tema: desde James S. Ackerman hasta Donata Batilotti, Mario Piana o el propio Howard Burns. El mismo Palladio Museum ha sido diseñado para cambiar al paso que la investigación avanza y ser contado todo ello de mano de sus protagonistas, los propios investigadores de la arquitectura de Andrea Palladio. No obstante la presencia de estos grandes estudiosos el proyecto expositivo no se 


\section{p̈h85 parasane}

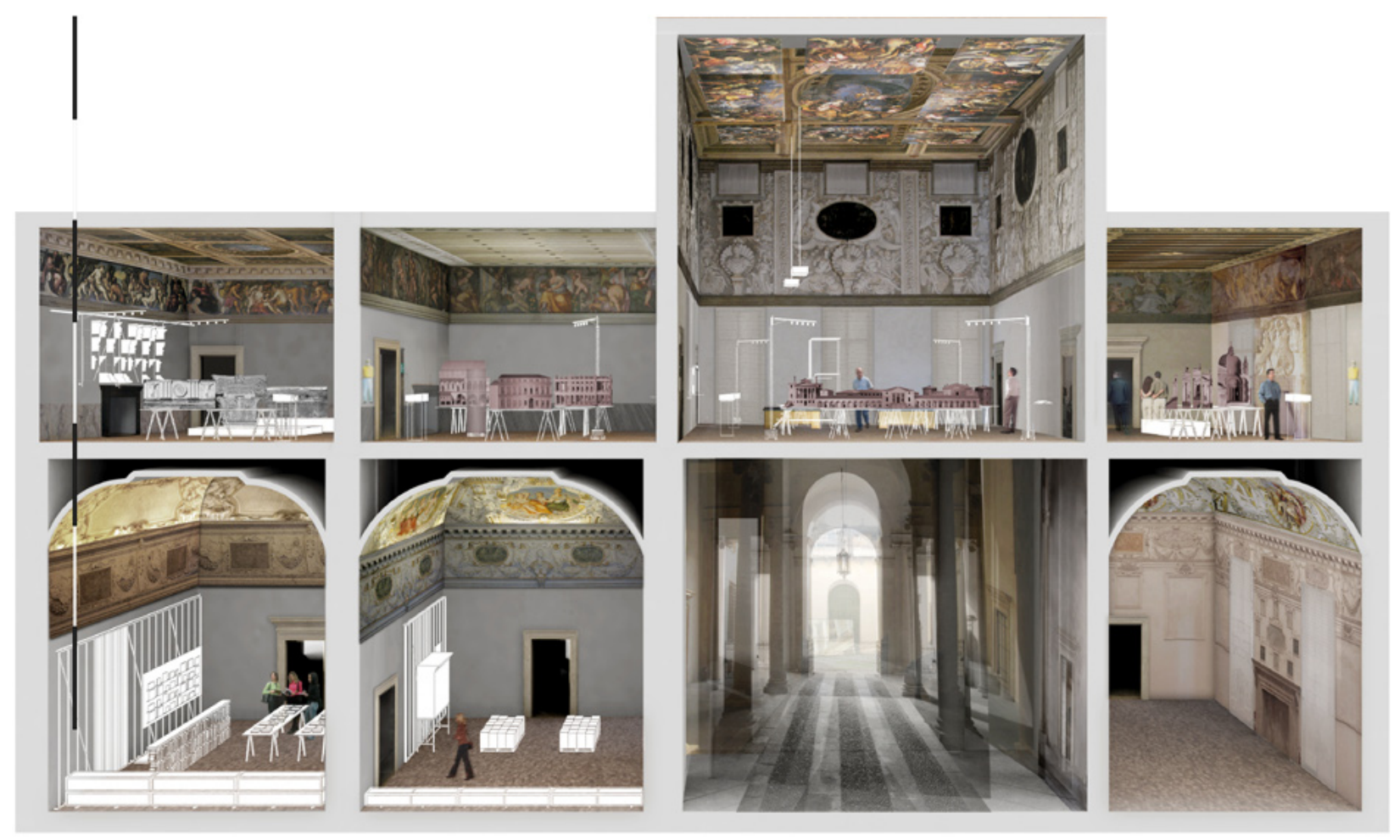

El Palladio Museum en el palacio Barbarano, Vicenza. Proyecto museográfico: Studio Scandurra, Milán | infografía Studio Scandurra, Milán

cierra a una gran especialización, sino que se abre al gran público a través de un proyecto divulgativo ambicioso y maduro, fruto de la gran experiencia pluridecenal del centro en la valorización y comunicación de la arquitectura palladiana.

\section{Recorrido a través de la obra de Palladio}

La primera obra introducida en el proyecto museográfico es el propio palacio, cuya visita completa ha sido ampliada y enriquecida para su más consciente interpretación. Tras admirar la riqueza del ritmo plástico de la fachada, el recorrido continúa en el amplio atrio tetrástilo abovedado, el espacioso patio con su gran porticado de órdenes superpuestos, que da paso, en su galería inferior, a la gran escalera que nos conduce a la planta noble, donde se desarrollará el recorrido expositivo interior. A día de hoy, y durante el año inaugural, el proyecto museográfico se compone de cinco salas, muy relacionadas con algunas de las más novedosas lecturas historiográficas de los últimos años: como la profunda investigación sobre las vivaces actividades económicas, sobre todo en torno al comercio de la seda, de los comitentes de Palladio, o la nueva visión de las villas palladianas como centros de producción agrícola y control territorial además de como simples locus amoenus.

Las salas están articuladas entre sí a través de la habitual prosecución armónica y serena que es propia de los apartamentos nobles de la arquitectura palaciega; en este caso, la planta noble se divide en distintas salas: la Sala del Libro, dedicada de hecho a la fortuna editorial de I Quattro Libri dell'Architettura y a la villa Rotonda; la Sala della Pietra, que enfoca las tecnologías ligadas a la construcción en piedra; la Sala della Seta, dedicada a Vicenza vista desde la agilidad en los negocios de los comitentes y las familias que son comitentes de Palladio. La Sala del Grano e della Gloria concentra su atención en una tipología muy desarrollada por Palladio como es la villa y que resume toda una política de la República de Venecia de revalorización de la terraferma veneciana. Esta sala expositiva se encuentra en el salón de honor del palacio, el cual conserva la decoración original que- 
rida por el comitente, Montano Barbaro, y que recupera, en su rico ciclo decorativo del mismo modo que las villas de Palladio en el territorio véneto, la cultura de la antigua Roma. La última sala permanente de este primer diseño expositivo está dedicada a la ciudad de Venecia, donde Andrea se traslada en su madurez para realizar algunos de sus proyectos más importantes y ambiciosos. La muestra se concentra, sin embargo, sólo en uno de ellos: la iglesia del Redentor en la Giudecca.

Por último, la sala de exposiciones temporales hospeda hoy, con un proyecto expositivo sabiamente introducido en el aparato decorativo de época de la sala, la exposición Home Sweet Home, donde 17 estudiosos de Palladio describen su obra palladiana preferida junto a las razones de su elección.

El recorrido goza de la presencia de maquetas de obras palladianas, como la del propio Redentor o de la Loggia del Capitanato, modelos a escala natural de elementos de construcción en piedra, como capiteles y arquitrabes, pero también libros en ediciones preciadas, siendo todos estos fondos la colección más importante del centro. A ellos se unen dibujos autógrafos de Palladio que enriquecen el discurso expositivo y que rotarán de acuerdo al proyecto museográfico.

Siendo el mismo edificio parte integrante de la exposición, el allestimento proyectado respeta la musculatura arquitectónica y la decoración original del palacio y las salas, en las cuales el mobiliario diseñado se posa ligeramente sobre los diferentes ambientes o se funde armónicamente con ellos, como las proyecciones virtuales sobre las paredes o los elementos que contienen las instalaciones audiovisuales. El diseño expositivo apuesta decididamente por una estética donde la tecnología y los expositores se funden con una sensación de taller de trabajo, un museo-taller, un workshop donde los objetos que observamos son ellos mismos herramientas para producir nuevo conocimiento y que involucran conjuntamente, esta vez, a investigadores de consolidada trayectoria científica con el gran público, que se siente así introducido en la continua investigación que se lleva a cabo en el CISA sobre la arquitectura de Andrea Palladio.

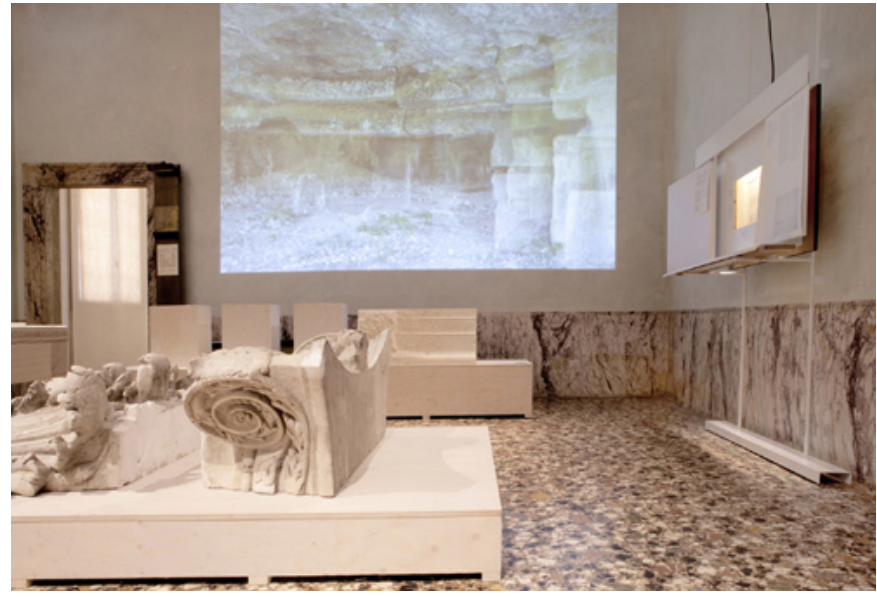

El Palladio Museum en el palacio Barbarano, Vicenza. Sala delle Pietre.Proyecto museográfico: Studio Scandurra, Milán | foto @ C Filippo Romano

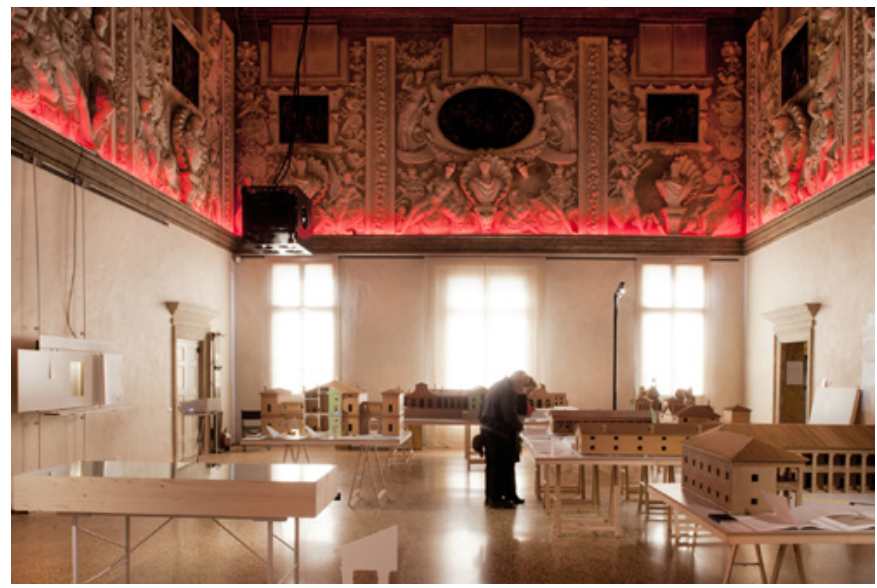

Sala del Grano e della Gloria | foto (c) Filippo Romano

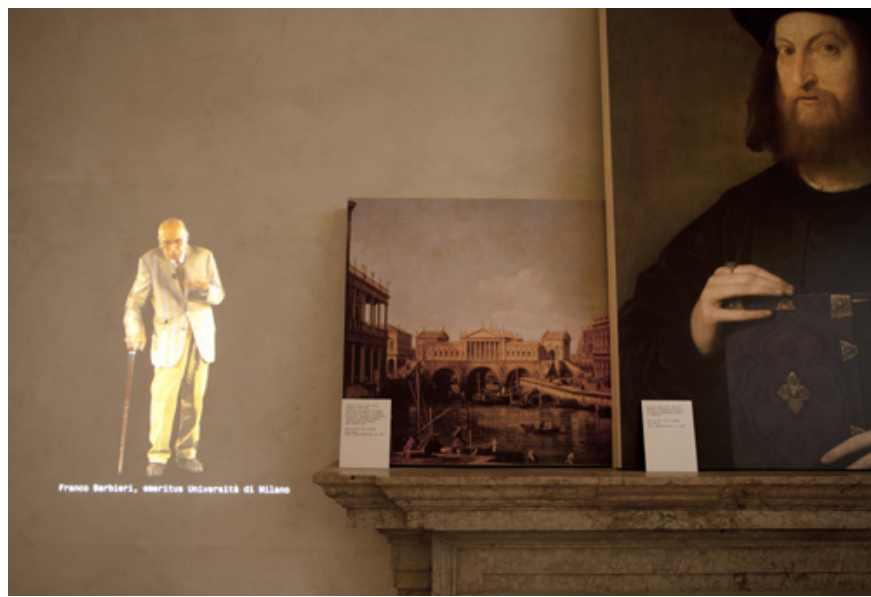

Sala della Carta | foto (c) Filippo Romano 